Cahiers de recherches médiévales

\title{
Quand le monde entre dans la chanson
}

Chansons politiques, chansons de croisade, serventois et autres tensons de trouvères

\section{Marie-Geneviève Grossel}

\section{(2) OpenEdition}

1 Journals

Édition électronique

URL : https://journals.openedition.org/crm/1863

DOI : $10.4000 / \mathrm{crm} .1863$

ISSN : 1955-2424

Éditeur

Honoré Champion

\section{Édition imprimée}

Date de publication : 15 décembre 2004

Pagination : $213-230$

ISSN : $1272-9752$

\section{Référence électronique}

Marie-Geneviève Grossel, "Quand le monde entre dans la chanson », Cahiers de recherches médiévales [En ligne], 11 | 2004, mis en ligne le 10 octobre 2007, consulté le 15 décembre 2022. URL : http:// journals.openedition.org/crm/1863; DOI : https://doi.org/10.4000/crm.1863

Ce document a été généré automatiquement le 15 décembre 2022.

Tous droits réservés 


\section{Quand le monde entre dans la chanson}

Chansons politiques, chansons de croisade, serventois et autres tensons de trouvères

\section{Marie-Geneviève Grossel}

$1 \mathrm{Au}$ commencement étaient les troubadours, au commencement était la canso. Ce double postulat, sorte de vérité dogmatique que l'on pourrait mettre en exergue à tant d'études modernes, sera mon point de départ que, mon sujet oblige, je vais tout de suite m'efforcer certes pas de contredire, mais d'assortir de toutes sortes de nuances.

2 Tout d'abord au sujet des troubadours; ils ne concernent pas mon propos puisque je vais évoquer les trouvères. Néanmoins le fait incontestable qu'ils aient précédé dans le temps les trouvères n'est pas - ou plutôt ne devrait pas être - synonyme absolu de précellence ; car raisonner en terme de hiérarchie valorisatrice (comme on le fit jadis pour les Latins par rapport aux Grecs) ne paraît pas la démarche heuristique la plus féconde. Au Moyen Âge, tout art se veut imitation et c'est dans et par l'imitation que se crée l'originalité. Je fais miennes ici les fines analyses de Suzanne Thiolier, spécialiste des poètes d'oc s'il en fut, sur ce sujet : l'œuvre est le résultat d'une tension qui se crée chez le poète dans la nécessité où il se trouve en raison de ce qu'est l'esthétique médiévale, nécessité de concilier les exigences des topoi avec sa propre liberté intérieure. C'est cette tension même qui génère la poésie, l'expression de l'énergie poétique, car une règle imposée n'a jamais nié la liberté individuelle, mais elle la contraint au contraire à se révéler ${ }^{1}$.

3 Ces très justes remarques, valables pour tous les poètes médiévaux, concernent-elles uniquement la canso, le Grand Chant, la chanson d'amour? Pas du tout, elles concernent toutes les productions lyriques, puisqu'en terre d'oc, le sirventes, s'il appliquait une esthétique de la rupture, était non moins étroitement codifié.

4 Commençons donc, pour respecter la chronologie, par rappeler ce que recouvrait la notion de sirventes. On sait qu'il s'agit d'un genre registral abondamment pratiqué par les troubadours ${ }^{2}$. Né dans le XII ${ }^{\mathrm{e}}$ siècle, il prend de l'importance au XIII ${ }^{\mathrm{e}}$ puisque le sirventes traite aussi de politique et que les troubadours de ce temps-là se trouvèrent 
étroitement engagés dans les horreurs de la guerre albigeoise, suivies, ce qui toucha non moins la poésie, par la mainmise des Français sur des provinces où ils allaient imposer leur langue, leurs lois, leur administration et leurs ministériaux, tout cela au détriment des cours provençales dans lesquelles la lyrique avait trouvé son origine et son plein épanouissement. Le sirventes a été défini par A. Jeanroy comme «des chapelets d'injures dénuées de tout atticisme $»^{3}$; mais l'on sait que le très grand savant médiéviste, en homme de son époque, s'offusquait du moindre manquement à la politesse et lisait tout texte poétique avec les lunettes du Romantisme. Si l'on veut plutôt se référer à une définition presque contemporaine des poètes, voici ce qu'en disent les Leys d'amors (1388):

Le sirventes doit traiter de blâme, de reproches généraux pour corriger les fous et les méchants ; on peut aussi traiter si l'on veut du fait de quelque guerre. ${ }^{4}$

5 Se fondant sur la définition médiévale, les Modernes subdivisent habituellement les sirventes en sirventes personnels, sirventes moraux et sirventes historiques/politiques. Dans le sirventes personnel, on attaque un ennemi en toutes ses faiblesses, on rapporte diverses anecdotes scabreuses de son existence, on caricature son physique, on fait des gorges-chaudes sur sa pauvreté, ou sa vile naissance, et, bien sûr, on souligne sa nullité lyrique ; on comprendra que, souvent, ces textes soient grotesques, voire orduriers; mais on trouve aussi des sortes de "critiques littéraires ", puisqu'un rival est sur la sellette. Pour se dédouaner peut-être de ce que le genre charriait de bassesses, on le nommait également sirventes joglaresc. À nos yeux, assurément, les meilleurs sont les galeries de portraits ridicules.

6 Le sirventes moral est d'inspiration beaucoup plus sérieuse et austère. On en trouve surtout au XII siècle. Il s'agit de donner des leçons aux contemporains qui sont topiquement lâches, bêtes et méchants, par opposition à un passé plus pur et valorisé. On trouve dans cette catégorie des textes où se posent les plus graves questions qui pouvaient tourmenter une époque, le destin, la liberté, la générosité, l'authenticité ou l'affaiblissement de valeurs ardemment cultivées, y compris poétiques.

7 Quant aux sirventes politiques, on y range les chants de guerre, appel à la rescousse ou à la bataille ou au courage des belligérants; on y critique avec véhémence rois, gouverneurs et, avec prédilection, le clergé. De tels textes sont souvent de vraies petites machines de guerre, en une époque où l'on sait déjà ce que veulent dire propagande et opinion publique.

8 À côté de cet aspect thématique du genre, il faut aussi s'attacher à ses caractérisques formelles, puisque séparer les deux est une véritable erreur. Le point de départ, essentiel, est le mot lui-même sirven, avec toutes les questions qu'il soulève. Issu du verbe signifiant servir, le terme avait d'abord été défini comme poème composé par un serviteur pour un maître qui le lui avait demandé; mais, de nos jours, on s'accorde à penser que le sens véritable du mot est celui qu'en donne la Doctrina de compondre dictatz, traité rhétorique en occitan ${ }^{5}$, datant de la fin du XIII ${ }^{\mathrm{e}}$ siècle : le sirventes s'appelle ainsi en raison du caractère servile d'un poème qui se soumet à un mètre utilisé antérieurement. Or on sait que le pretz d'une chanson vient en partie de ce que le troubadour a inventé à la fois la mélodie et le schéma rythmique-rimique de son texte. Nous retrouvons ici Suzanne Thiolier qui insiste sur l'esthétique de la rupture cultivée par un registre, où s'opposent une mélodie et une structure formelle venues d'ailleurs, et un texte personnel original; partant, jamais un tel texte ne saurait prétendre à une 
harmonie entre ses composantes, la valeur naît cette fois de la tension qui se crée entre les deux, que la canso posait, par définition, comme intrinséquement liées.

D'un point de vue pratique, le compositeur de sirventes prend bien soin de choisir une mélodie dont la popularité contribue à la rupture esthétique, tout en apportant le succès à son texte par une facile mémorisation. Quoique le troubadour qualifie son poème de "chanson engagée, facile et vulgaire ", il n'empêche que certains sirventes ont survécu, parfois des années durant, aux raisons qui les avaient fait naître et cela même dans le cas de sirventes politiques, nécessairement les plus dépendants du temps qui passe et fait tout oublier. Il est vraiment étonnant que certains textes nous soient parvenus, les manuscrits, dont beaucoup proviennent de l'Italie du XIV ${ }^{e}$ siècle, les ayant sans doute recueillis par voie orale (avec toutes les altérations que cela impose) ou sur des rotuli, ces fragiles « feuilles volantes » que les jongleurs gardaient par devers eux pour se rafraîchir la mémoire.

Ces remarques, aussi générales que dénuées d'originalité, m'amènent à boucler la boucle qu'ouvrait le postulat liminaire " au début était la canso... ». Car par définition, la canso est intemporelle, transcende le temps et passe donc ${ }^{6}$, quasi immuable, de l'oral à l'écrit, sans avoir subi l'usure des événements; au contraire, le sirventes est fragile, temporaire, fugace; il se perd aussi vite qu'il se crée; en outre, considéré comme de peu de valeur, il n'était naturellement pas promis à la mémoire de l'écriture, pour nous seule susceptible de le faire encore connaitre. On se souviendra enfin de la crainte exprimée par maints héros épiques de se voir taxés de lâcheté et de connaître le déshonneur que suscitent les males chansons. Que serait-ce donc qu'une male chanson, sinon un sirventes? On peut donc conclure qu'à un moment précis de l'histoire, les troubadours ont recouru, pour composer leurs males chansons politiques ou critiques, au modèle de la canso, avec tous les avantages que l'on peut concevoir de cette piquante innovation, avantages esthétiques, essentiels pour des artistes et des publics aussi raffinés que ceux des pays d'oc. Mais avant de mériter le terme technique de sirventes, le registre thématique existait déjà, pratiqué sous d'autres formes qui ne nous sont généralement pas parvenues, sauf les moralités en latin, écrites ou dédiées à des clercs par d'autres clercs, ces maîtres tout puissants de l'écriture.

11 Je quitte ici les terres ensoleillées des troubadours pour retrouver au Nord mes trouvères. À vrai dire, si beaucoup de critiques récents se sont occupés de la canso d'amour, le sirventes a suscité moins d'intérêt, mis à part les passionnants travaux de M. Aurell et de S. Thiolier, ainsi que diverses remarques dans des ouvrages de D. Rieger 7 . Depuis les grandes thèses, déjà anciennes, de R. Dragonetti et de P. Zumthor, les travaux sur les trouvères ne se sont pas vraiment multipliés. Dans sa typologie lyrique, $\mathrm{P} . \mathrm{Bec}^{8}$ répartissait la production des trouvères selon un double critère: « registre aristocatisant vs registre popularisant » et «textes imitant les œuvres en langue d'oc vs textes ne les imitant pas ». Dans le registre popularisant se classent les chansons à danser, les aubes et les pastourelles, -en tenant compte de très nombreuses interférences registrales- puis les chansons "de femmes", d'ami(e)s ou chansons de toile; dans le registre aristocratisant, outre la canso, on trouve les chansons de croisade et les sottes chansons. Mais on chercherait en vain le serventois (pourtant aisé à définir comme « copie » du sirventes), et l'on ne sait trop où ranger les chansons que Jeanroy regroupait sous le label «satiriques et bachiques", ou encore ce que Leroux de Lincy appelait pour sa part «chansons historiques ", tous textes que ne caractérise pourtant pas leur rareté. Il est, précisons-le, hors de doute, qu'il s'agit bien là de textes lyriques 
au sens technique du terme, de textes qui furent chantés. Se pose ici le problème de trouver à ces «chansons du siècle » une place en typologie : appartiennent-elles à la catégorie «imitation des pièces d'oc»? Sont-elles "popularisantes » ou « aristocratisantes" ? Et, pour trouver un terrain plus sûr, le Moyen Âge lui-même les a-t-il désignées sous un terme dont nous pourrions user?

À côté du mot d'oc sirventes, le terme serventois est bien attesté et, cette fois encore, ce sont les spécialistes des troubadours qui en ont suivi avec patience la génèse et les variations, bien que cette étude fût, en fait, annexe à ce qui faisait leur recherche. Dans sa thèse (1973) Suzanne Thiolier et, un peu après, D. Rieger, signalent que les dictionnaires définissent serventois comme "poésie lyrique/plaisanterie/discours ", le Godefroy spécifie "pièce de vers ". Le mot est déjà présent dans le Roman de Rou de Wace ${ }^{9}$ où l'on notera la rime significative gabeis/serventeis et dans le Roman de Thèbes :

A ceus dehors gabent et rient

Et por escharz serventois dient. (v. 1559-60) ${ }^{10}$

L'éditeur indique "plaisanterie »; dans la Chevalerie Ogier, c'est le sens de "discours » qui l'emporte : « Li dus respont, n'i fist lonc serventois » (v. 11177) ${ }^{11}$.

Mais dans le domaine de la chanson lyrique, il faut noter la rareté du terme, en même temps d'ailleurs que sa technicité: «Mout liement dirai mon serventois [...]»; ainsi commence une chanson ${ }^{12}$, qui, bien que répertoriée sous le nom de Moniot d'Arras, n'est pas de lui, mais représente un cas bien spécifique de chanson politique sur la victoire de Taillebourg (1242) : écrite à l'évidence par un partisan des «Français de France » contre les Anglais du roi Henri III et aussi leurs partisans comme le comte Hugues de Lusignan, c'est bien une male chanson où l'on se gausse nommément de vaincus ridiculisés, on peut donc déduire qu'ici, le choix du mot « serventois » aurait été influencé par le terme, bien connu à cette époque, de «sirventes». Mais c'est une pièce tardive.

On sait d'autre part que le terme se généralise dans le second Moyen Âge, en même temps qu'il change totalement de signification: on le trouve alors dans les grandes cités du Nord, comme Arras, Valenciennes ou Amiens, où il désigne une pièce couronnée par les Puys sous la désignation de "serventois couronné ». Il s'agit désormais de poésies pieuses adressées à la Vierge, ce qui n'a évidemment plus rien à voir avec la présente recherche. Les philologues eux-mêmes, s'ils sont d'accord sur l'évolution du mot et de sa thématique, n'ont pas réussi à trancher de façon incontestable sur la date de son apparition première, en terre d'oc ou en terre d'oïl; dans les deux zones linguistiques, le mot semble bien s'apparenter au verbe "servir", et la nuance de « discours » paraît plus marquée au Nord qu'au Sud, puisqu'elle y est constante dans l'épopée et les premiers romans ; la racine du mot aurait-elle été la source, au Sud, de l'idée novatrice de faire de ce « discours au service d'une cause » un « discours asservi à une canso " ? On notera qu'un ménestrel comme Jean de Condé (qui n'avait sans doute pas lu la Doctrina de compondre dictatz) sait parfaitement gloser "serventois" par "servir $»^{13}$. Bref, on peut sans préjugé penser que, pour que qui est du genre des males chansons, ni les troubadours ni les trouvères n'avaient besoin d'un initiateur pour composer en ce registre; quant au terme de "serventois» appliqué à la lyrique, sa rareté même prouverait qu'il peut s'agir d'une allusion du trouvère qui l'emploie à des réussites jugées exogènes et prestigieuses; ce qui n'empêche pas qu'il existe un très grand nombre de pièces que ni les manuscrits ni les trouvères n'ont pensé à classifier et 
qui s'apparentent pour la thématique à ces « chansons du siècle » sur lesquelles je me penche ici.

Nous trouvons, à côté de pièces proprement politiques, des chansons satiriques (sur le siècle, les femmes, les ordres etc.) et des chansons morales. Par contre, chez les trouvères, il n'existe pas de galeries de portraits littéraires; on pourrait cependant rapprocher les sirventes joglaresc des jengles et contregengles ${ }^{14}$ qui, précisément, font bien attention de mettre en scène des jongleurs. Les textes sont en effet caractérisés par leur grossièreté (relative), la bassesse des attaques (sur la famille du rival plus que sur son origine puisque le texte pose topiquement l'auteur comme vil), enfin le recours à l'injure lorsque les arguments se font un peu courts. Cependant les jengles ne sont pas (ou presque pas) des textes chantés, ils se rangeraient plutôt dans la vaste rubrique, difficile à déterminer, du dit, seule la thématique ici serait commune. D'autre part la définition du narrateur-trouvère comme jongleur empêche toute critique sur sa valeur de compositeur puisqu'aussi bien au Moyen Âge, se dire «jongleur » implique qu'on ne se dise pas "auteur", mais uniquement voix qui proclame le texte d'un autre, ou l'histoire d'un autre temps. Les narrateurs des jengles se moqueront donc de l'ignorance de l'adversaire, non de sa valeur, le rival a un "bagage littéraire " négligeable ou encore il mélange tout et chante de «Gauvain le couard» ou du "Sarrasin Charlemagne ». D'autre part, il arrive qu'un trouvère se définisse comme " un pauvre " un peu à la façon d'un Rutebeuf, ou d'extraction humble, en tout cas plus humble que la dame chantée et exaltée. Mais il est difficile de déterminer si c'est là un élargissement de la topique originelle du parage de la bien-aimée ou si cela repose sur quelque trait réel ; quoi qu'il en soit, on ne trouve ce motif que dans le registre du Grand Chant, non pas dans les serventois.

Quant à critiquer de l'intérieur la thématique courtoise, les trouvères ont choisi de le faire dans la parodie, avec la sotte chanson. Les troubadours n'ont pas cultivé ce genre pour une raison aussi simple que tragique, comme le souligne S. Thiolier avec bon sens : pour parodier un registre, il faut que ce dernier accuse quelques signes de fatigue dus à une trop longue durée, durée qui fut refusée à l'art des troubadours, pour les tristes raisons que l'on sait ${ }^{15}$.

18 J'éviterai donc de parler de serventois pour les quelques exemples dont je vais à présent examiner la thématique, au profit de termes, plus généraux, mais moins réducteurs, comme chansons politiques ou historiques ; je laisserai de côté, cette fois, les chansons dites "satiriques", mises à part quelques exceptions ${ }^{16}$. C'est une catégorie bien représentée, surtout dans les cercles du Nord, volontiers didactiques, elle participe terriblement à nos yeux du lieu-commun, contre Amour, contre l'avarice des nobles etc.; les critiques contre le clergé qui évoquent le plus les sirventes des troubadours sont, je crois, les chansons de Rutebeuf, mais il semble vraiment difficile de parler de Rutebeuf comme d'un " trouvère ", au sens lyrique du terme.

Le monde entre dans la lyrique d'oïl de deux façons : du côté de la politique religieuse, ce sont les chansons de croisade; du côté de la politique profane, ce sont les chansons historiques. S'y ajoutent des cas particuliers, celui d'Arras, cité-cible des critiques et des louanges de ses poètes, souvent des humbles; ceux de Philippe de Novare, Thibaut de Champagne ou encore Colin Muset - tous de véritables personnalités littéraires, méritant chacune quelques mots.

20 La chanson de croisade représente l'exemple par excellence d'un genre où interfèrent les registres ${ }^{17}$, ce qui explique qu'elle s'insère assez mal dans le cadre d'une typologie. 
Son caractère remarquable est qu'elle est le seul genre lyrique à son époque qui soit thématiquement lié à un événement précis, souvent repérable dans le temps et dans l'espace : elle est donc à proprement parler une chanson " de circonstances ». Le Je qui s'y exprime est celui qui appelle, celui qui part, celui qui évoque un événement récent (défaite ou prêche) justifiant le nouveau départ, le Je est celui du monde d'ici que symbolise la douceur de l'amour tandis que se dresse en face l'ailleurs de l'Outremer, terre d'épreuve et terre de sanctification. La chanson se joue entre ces deux réalités antithétiques dont aucune n'est moins vraie que l'autre. Bédier et Aubry ${ }^{18}$ ont pu, de façon très légitime, classer selon la chronologie les vingt-neuf pièces qu'ils incluent en leur anthologie et qui s'échelonnent entre 1147, date de la deuxième croisade, et 1248, qui est celle de la septième et avant-dernière. En effet, la première croisade ne nous a pas transmis de chanson, mais qu'elles aient existé est confirmé par le terme générique de chanson d'outrée sous lequel elles sont désignées dans les textes. Le témoin unique qui nous reste de la croisade prêchée en 1146 par Bernard de Clairvaux et conduite par Louis VII et Conrad III est un texte anonyme avec un refrain d'allure très popularisante. La troisième croisade, celle de 1189-1191, où s'illustrèrent Barberousse, PhilippeAuguste et Richard Cœur de Lion, est la plus représentée avec ses neuf chansons. P. Bec interprète cette richesse par l'influence " désormais contraignante $»^{19}$ de la lyrique d'oc sur celle d'oïl. Mais peut-être faudrait-il aussi penser au fait, bien attesté et vérifié, que la troisième croisade est la seule ${ }^{20}$ à avoir eu en terre d'oïl un statut proprement littéraire, à avoir généré des œuvres, plus ou moins contemporaines, et cela dans toutes les formes d'écriture alors pratiquées. En effet, si la croisade de $1204^{21}$ et celle de 1219 nous ont laissé chacune deux chansons, le mérite en revient à Hugues de Berzé qui en a écrit une à chaque fois; de même, la croisade des Barons en 1239 dont l'importance historique est des plus restreintes, nous transmet sept chansons, mais quatre sont de Thibaut de Champagne et deux de poètes de son cercle. Enfin parmi les trois chansons traitant de la croisade de 1248 , l'une est probablement de Joinville. Quelques chansons anonymes, souvent de facture très simple, ne présentent pas d'indications permettant de les dater. Mais leur caractère propangadiste et les irrégularités que souvent elles manifestent semblent indiquer qu'elles furent l'œuvre de jongleurs ou de poètes à gages qui accompagnaient les grands seigneurs, comme le montre l'exemple d'un Guiot de Provins. Chantées et colportées de ville en ville, ces pièces gardent la trace de leur existence mouvementée et les difficultés que rencontre l'éditeur à établir la succession des rimes, des strophes, voire des idées est l'indice d'une transmission orale où les textes ont naturellement souffert.

21 La plus ancienne des chansons de croisade, "Chevalier mult estes gariz", est accompagnée d'une musique en cursive de la fin du XII siècle qui n'est pas sans poser de difficiles problèmes d'interprétation aux musicologues. Le ton est marqué par un enthousiasme sensible que souligne le retour du refrain :

Ki ore irat od Loovis

Ja mar d'enfern n'avra pouur

Char s'alme en iert en pareis

Od les angles nostre Segnor. ${ }^{22}$

La chanson opère un précieux recensement des derniers événements, cause de sa naissance : prise d'Édesse (appelée Rohais de son nom arabe er-Roha) par les Chanelieus (les Cananéens ou païens) et leur chef Sanguin le Felon, le fameux Zenghi dont le nom roman, altéré, est évidemment des plus symboliques. La thématique, qui deviendra obligée, est la même que celle développée par les chansons de geste, dites, elles aussi, 
"chansons de croisade » : la terre où Dieu souffrit sa Passion est «sa terre » au sens féodal du terme; les Infidèles font des églises les étables de leurs bêtes; suivons l'exemple du roi (Louis VII) qui a quitté le vair et le gris, laissé toute sa richesse et son royaume, nous gagnerons en échange le Paradis.

À étudier la musique des autres chansons, P. Aubry ne tire pas la conclusion que les poètes d'oïl aient utilisé une mélodie préexistante comme le voulait le sirventes. Pourtant le jeu sur les interférences registrales n'est pas sans évoquer une certaine esthétique de la rupture, cultivée par divers moyens, ainsi dans cette chanson de croisade anonyme (III ${ }^{e}$ croisade sans doute) qui commence résolument à la façon d'une aube :

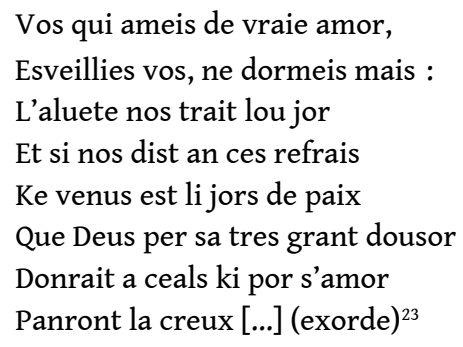

La chanson tire, d'autre part, abondamment parti de l'image courtoise du service d'amour, impliquant ici la réciproque, puisque l'ami est Dieu, qui donna sa vie pour ceux qu'il aimait. Mais nous retrouvons la parénétique avec l'affirmation : "Nos n'en avons poent de demain. (v. 41) ». Mieux vaut mourir aujourd'hui dans la gloire que tout-à-l'heure, en misérable.

Un groupe de trois chansons, toujours de cette même croisade de 1190, s'apparente d'autre façon aux sirventes et non pas aux chansons de croisade occitanes, également bien attestées : elles ont été échangées entre (ou plutôt elles prennent pour cible) deux grands trouvères de l'époque : le premier est Hue d'Oisi, dont il ne nous reste, outre la chanson en question, qu'un Tournoi de dames, sans doute lui aussi polémique sous ses apparences anecdotiques et flatteuses; le second est Conon de Béthune, seigneur rendu célèbre par la chronique deVillehardouin. Là encore les circonstances historiques nous sont bien connues : la prise de Jérusalem, après le désastre de Hattîn qui avait anéanti le Royaume latin d'outremer, avait soulevé en Occident une vague d'émotion extrême ; aussitôt les rois de France et d'Angleterre, pressés par le Pape, avaient pris la croix. Mais en fait ni l'un ni l'autre n'avaient entamé le moindre préparatif: Henri II Plantagenêt, vieilli, malade, était en butte à la rebellion de ses fils et de son épouse, tandis que Philippe-Auguste fut toujours plus intéressé par son royaume que par la croisade. Le départ fut plusieurs fois remis, d'abord du fait de la mort de la reine de France, Isabelle de Hainaut, puis ce fut celle de Henri II lui-même, auquel succéda son fils Richard. En face de ces atermoiements, Conon de Béthune, bien connu pour ses liens avec le parti flamand puissant à la cour, s'enflamme et recourt à sa vièle pour prêcher haut et fort la croisade et ses prouesses, il souligne - preuve sans doute qu'existaient déjà des opposants à la croisade en armes - que les femmes n'en aimeront que mieux les guerriers à leur retour, ou cultiveront pieusement le souvenir des héros. Mais, il faut le croire, ces propos pleins d'ardeur de notre trouvère ne furent pas non plus suivis d'un effet personnel; et puis le parti flamand avait perdu de sa suprématie avec la mort de la reine... Hue d'Oisi, qui avait été "maitre en l'art de trouver" du bouillant Conon, prend à son tour la vièle et ne se prive pas de railler le beau-parleur. On remarquera la virulence de son ton et l'attaque contre le roi failli qui est sans doute 
Philippe-Auguste! Hue d'Oisi était fieffé au Nord, comme Conon, et en Brie par son épouse. Il est probable que ces textes, remplis d'invectives, recèlent plus qu'une simple moquerie pour un départ retardé. Conon répondit avec moins de fureur qu'on eût pu le craindre ${ }^{24}$. Il tire même un petit coup de chapeau à son maître- où il faut sans doute voir bien de l'ironie...

Or ai je dit des barons ma semblance.

Se lor en poise de ce que je le di,

Si se preignent a mon maistre d'Oisi

Qui m'a apris a chanter des enfance. (v. 49 sq.) $)^{25}$

Aux antipodes de cette querelle entre trouvères de haut vol, on peut situer la chanson de "Maître Renaut ${ }^{26}$ avec sa versification aussi simple que les chansons «folkloriques»: deux rime en $a, b$ masculines et un refrain $c c$ avec deux rimes féminines, soit $a b a b a b a b c c$. Les coblas sont singulars. On rappellera que les troubadours boudaient le refrain considéré comme trop populaire, y compris pour les sirventes. Ici le ton frôle « tantôt le sermon, tantôt la complainte », l'auteur, sans doute un clerc comme l'indique son titre de "maître », était cependant d'humble origine et très proche des croyances des « pauvres gens » dont nous entendons si rarement la voix en notre lyrique. Sa langue indique qu'il venait du Nord Est de la France.

La chanson de croisade est l'une des chansons lyriques où se discerne le mieux la personnalité littéraire de l'auteur. Ainsi des vers mélodieux et tendres du Châtelain de Couci, chantant le départ pour une croisade dont il ne devait pas revenir, avec toute la rebellion d'un amant qui, il l'avoue pudiquement au détour du vers, ne fut pas toujours repoussé. La révolte, habituellement réservée aux chansons dites de femmes, éclate ici dans l'apostrophe multipliée à Dieu, jusque trois fois dans les derniers vers de la dernière strophe, un Dieu dont le visage implacable prend presque les traits du destin, et qui se résout en l'ultime «à Dieu » dont on sait qu'il se réalisa tragiquement avec la mort en mer du poète ${ }^{27}$. Que le Moyen Âge ait été sensible, et durablement, à ces vers, nous le prouvent, outre le lointain roman qu'en tira Jakemes à la fin du même siècle, les très nombreuses réminiscences de ces vers dans les chansons d'aventures au $\mathrm{XIII}^{\mathrm{e}}$ siècle $^{28}$.

S'il est vrai que beaucoup de chansons de croisade, surtout celles des grands trouvères, interfèrent largement avec la chanson d'amour, (partir outremer devient essentiellement quitter la dame), c'est dans un autre registre que les poètes vont chercher leur thématique de pélerins en partance. Ils ne mettent pas en scène comme Rutebeuf une disputoison du croisé et du décroisé, mais ils incarnent en un Autre le refus de mourir et la peur hideuse de l'Infidèle, amplifiée par la mythification épique. C'est pourquoi certaines chansons de croisade, naturellement écrites par des hommes, sont devenues des «chansons de femme ", la femme représentant alors le côté sentimental de l'homme, qui peut ainsi accéder à la plainte sans fausse honte ni déshonneur. Il semble même que, sous ce couvert, certains poètes aient ainsi prêté leur voix à d'authentiques sentiments féminins et nous fassent, là encore, entendre ce qui ne nous est pas facilement audible habituellement, la révolte et la douleur de celles qui restaient, les femmes ou les mères, promises aux longues heures de l'incertitude, du doute, d'un désespoir où le travail de deuil ne pourrait jamais se faire faute d'être sûr de l'irréfutable ${ }^{29}$. La chanson de Guiot de Dijon, écrite probablement dans les petites cours de la Champagne méridionale, se donne ainsi à entendre sous l'apparence " popularisante » de la rotrouenge. Elle est en réalité très subtilement construite et met 
en scène une toute jeune fille en pleine révolte, remplie d'épouvante devant le départ de l'aimé :

Dex ! quant crieront outrée

Sire, aidies au pelerin

Por cui sui espoentée

Car felon sunt Sarrazin.(ch.10, refrain)

Et non moins désespérée par son propre avenir, puisque ses parents, s'ils comprennent sa peine, n'en parlent pas moins déjà de la marier à un autre : impuissance de la femme devant sa parenté, déchirure d'une séparation que la vie en se poursuivant rend définitive; au milieu de cette mise en scène du réel, Guiot introduit deux éléments culturels, une citation de Bernart de Ventadour visiblement conçu comme le parangon, le référent littéraire de la passion d'amour, et une évocation brûlante de sensualité à l'amour physique avec le motif de la chemise de corps laissée en ultime cadeau. Cette allusion a sans doute pour rôle de souligner la féminité naïvement avide que la chanson de toile notamment pose en axiome, tout en amplifiant très fort le pathétique. C'est ainsi que cette chanson de circonstances qu'est la chanson de croisade se révèle en profondeur intimement et secrètement remplie de la plus authentique réalité, celle du sentiment ressenti.

Pour avoir une vue d'ensemble de ce registre et pour conclure, j'évoquerai rapidement la chanson écrite par « un pauvre sergent de l'armée » :

$\mathrm{Ne}$ chant pas que que nus die

De cuer lié ne de joios $[. . .]^{30}$

31 Ce trouvère ne savait pas trop rimer, mais il connaissait bien la cause de tant d'échecs, la discorde qui séparait les "hauts hommes " auxquels pour sa part il devait obéir. Écrite après le désastre de Gaza par un poète de l'Est, la chanson déplore la mort du Comte de Bar, justifiée sans doute par sa fole envaïe, non moins douloureuse cependant. Là encore ce texte, de médiocre valeur lyrique, déborde d'un chagrin si révélateur et si puissant que le Chroniqueur a préféré à de longs discours d'analyse la simple retransmission de ces vers parfois fautifs. Nous avons la chance de posséder, si l'on ose dire, l'autre "son de cloche" avec la récrimination de Philippe de Nanteuil, noble trouvère et ami de Thibaut de Champagne, fait prisonnier à cette même bataille de Gaza $^{31}$. La faute, selon lui, revient entièrement aux chefs, qui ne font pas libérer les prisonniers ; quant à la "fole envaie », Philippe, qui y participa, n'en a nul remords ni même, semble-t-il, nulle conscience. La chanson se veut cette fois planh, plainte sur les morts, cri de détresse des survivants captifs. On déplore la perte de tant de chevalerie, en chemin, on a oublié Jérusalem et le tombeau du Christ : la chanson de croisade est à nouveau la mise en scène des sentiments et du pathétique, le tumulte du monde qui s'y engouffre avec ses guerres, ses meurtres et ses dissensions permet comme un écho de nous renvoyer la voix du poète, aristocrate ou simple piéton.

On remarquera que les chansons consacrées à la première croisade de saint Louis (1248) tournent plutôt autour du «miracle " qui vit revenir à lui un roi qu'on croyait déjà mort. Deux textes semblent originaires de la même région, l'Ile de France, le premier avec de légers traits picards (on a proposé Pontoise où le "miracle" eut lieu); le second se caratérise par la grande subtilité de sa construction, mêlée à la naïveté de la thématique (la conversion des soudans) très proche des chansons de geste. C'est certainement cette inspiration qui explique ici le choix du mot «serventois" par le poète pour définir son œuvre : 
Un serventois, plait de deduit, de joie,

Commencerai [...] (v. 1-2)

Au riche roi qui France a en baillie

Va, serventois, ton message conter [...](v. 46-47) ${ }^{32}$

évidemment à la composition habituelle de la canso. On sait qu'une dernière chanson, écrite à Acre un peu plus tard, évoque précisément la délibération organisée par saint Louis, tout juste libéré après le versement de sa rançon. Les arguments du trouvère, qui veut rester outremer comme son roi, sont si proches de ceux développés par le texte de Joinville que G. Paris était fort tenté d'attribuer ce texte au chroniqueur ${ }^{33}$. Joinville, dont on a pu remarquer qu'il avait une vraie "manie de l'écriture » sortait, il est vrai, de bonne école puisqu'il est le contemporain et le compatriote des cercles de poètes autour de son suzerain, Thibaut de Champagne. Il n'emploie pas le terme de «serventois » bien que sa poésie présente tous les traits de la poésie politique. Mais Joinville a ici bel et bien utilisé un schéma formel et une mélodie qui préexistaient et qui s'avèrent avoir été ceux d'un jeu parti échangé par Thibaut de Champagne et un certain Baudouin ; le jeu-parti avait déjà lui-même repris sa forme à une chanson du Châtelain de Couci; et, surtout, ces mêmes schéma formel et mélodie avaient été utilisés par Hue de la Ferté, dans une chanson violemment pamphlétaire contre Thibaut, au moment de la difficile Régence de Blanche de Castille. On peut donc deviner ici tout un jeu entre les poètes, se reprenant l'un l'autre des airs qui avaient été, en leur temps, vraiment «à la mode » et avaient véhiculé, en véritables serventois les critiques les plus outrancières et insultantes ${ }^{34}$. Un tel exemple montre la difficulté à classer des chansons qui mélangent les registres avec une liberté aussi absolue.

Hue de la Ferté sera pour nous la transition parfaite qui nous mène, sans quitter les cercles de poésie champenoise, de l'Outremer et ses guerres religieuses à la très prosaïque ambition qui mouvait les Grands lors des guerres de succession au comté de Champagne ou à la tête d'un Royaume dont le prince était alors un enfant.

Il est loisible d'imaginer que la stature politique du personnage est ce qui a permis la conservation du bouquet de chansons politiques, écrites pour être chantées contre Thibaut de Champagne dans les années 1226-1230. On pourrait aussi induire de son goût bien connu pour la lyrique le choix de cette arme très particulière contre lui. Quoi qu'il en soit, les circonstances où ces textes furent écrits nous sont bien connues, notamment grâce à Joinville. Cette véritable campagne de calomnies en vers fut déclenchée par l'opposition que la noblesse manifestait envers la Régente Blanche de Castille, doublement coupable d'être une étrangère et d'être une femme. Pour assurer son pouvoir, la Régente s'appuyait sur l'Église, en l'occurrence le cardinal Romain de Saint-Ange, et, après diverses péripéties, sur le comte de Champagne Thibaut, qui lui était apparenté par sa mère, Blanche de Navarre. En face, il y avait, eux aussi liés par une parenté fort enchevêtrée, les seigneurs de Coucy, les Dreux-Braine et le bâtard légitimé de Philippe-Auguste, Philippe de Boulogne. La famille des Braine, possessionnée en Champagne et en constante rivalité avec les comtes, s'était depuis longtemps distinguée par son mécénat littéraire ; elle comptait vers la même époque un trouvère, Jean de Braine, mais c'est un autre poète qui prit sa vièle pour composer, Hue de La Ferté, seigneur de la Ferté-Bernard ; il marchait derrière Pierre de Braine, comte de Bretagne et avait été personnellement indisposé par les prétentions des Thibaudiens au comté du Perche, tombé en deshérence.

Cahiers de recherches médiévales, 11 | 2004 
ue possède un réel talent de pamphlétaire et la première de ses chansons dirigée essentiellement contre Blanche de Castille se fait remarquer par une constante ironie, non dénuée de finesse :

Pourtant les arguments ne sont pas d'une qualité irréprochable s'ils devaient toucher le public des barons : c'est France personnifiée qui se plaint de voir « la reine et ses drus » avilir la noblesse. L'attaque vise Gautier Cornu, qui «dédaigne les barons/Pour la gent d'Espagne ». La xénophobie se portait déjà bien. Aux clercs les églises, à la maisnie du roi, la politique : argument très en faveur dans la geste! La gent de femenie doit rester objet de méfiance, la parole est à ceux qui « savent armes porter ». Bientôt les insultes glissent au plus bas: Thibaut, soutien de la couronne, né après la mort de son père, est un bâtard, il est gros, felon, il s'y connaît en chirurgie, autrement dit, il a empoisonné le roi Louis VIII, de surcroît, c'est un lâche. Enfin, son entente avec la Régente est fielleusement glosée par des sous entendus obscènes. Blanche n'est-elle pas surnommée dame Hersent?

À côté des trois chansons écrites par Hue de la Ferté, une quatrième, anonyme ${ }^{36}$, évoque les mêmes événements et se présente comme un dialogue entre deux partisans des barons coalisés. Chacune des strophes commence par le prénom d'un des deux personnages, Gautier et Pierre, ce qui donne un air de jeu-parti ou de tenson à ce dialogue. Si dans un premier temps, les deux voix s'accordent pour se moquer de la lenteur des «barons » à agir, elles ne cachent pas leur parti : cette chanson est un appel à Philippe Hurepel, Pierre de Bretagne et son beau-frère le comte de Bar, à marcher contre les Basques, ce terme méprisant, qui regroupe la Castillane et le Navarrais, a un parfum d'épopée certainement recherché et souligne le goût de ces barons, amateurs de grands coups d'épée et prompts à confondre ambitions et prouesse... Toutes ces chansons politiques sont d'une facture simple et probablement construites sur divers modèles connus. Il semble bien évident qu'ici les motifs l'emportent sur l'art de les agencer. Le fait que ces chansons aient été conservées, alors que le parti des barons fut si bien vaincu qu'on préféra ne plus trop évoquer les faits, reste assez troublant et mystérieux. Une des possibilités, qui n'est pas la plus invraisemblable, pourrait être que Thibaut, doué d'intelligence et d'humour, avait su faire passer son goût pour la poésie avant son amour-propre.

Fait amusant, pour trouver un autre petit groupe de chansons politiques, il faut passer du côté de la cour. Louis IX était pourtant connu pour son refus austère de l'art du trouver profane. Mais ce rejet n'atteignit sa pleine force qu'après la mort de la reine Blanche qui sortait d'une famille de mécènes éclairés. C'est des années où elle vivait encore que date le poème sur la victoire de Taillebourg (avec un petit coup de chapeau aux Champenois...) et celui exaltant le retour du roi à la vie avant son vœu de croisé (1244). Ces deux chansons, nous l'avons vu, prennent le titre de serventois. 

s'élève ainsi contre le roi Henri III, coupable de vouloir lever une dîme sur son clergé ; nous savons que Henri III destinait l'argent à son frère Edmond auquel le pape avait accordé le royaume des Deux-Siciles contre le Hohenstaufen Manfred, ennemi notoire d'Alexandre IV ; mais les clercs se moquaient bien d'Edmond et refusaient d'être taxés ! Le texte est une longue suite de lamentations inscrites sous la bannière emblématique de Jérémie ; elle se clôt sur une solennelle adresse à Henri III qui doit « regarder » du côté de Louis (IX) s'il cherche un modèle. Cette chanson est écrite en anglonormand. Deux autres chansons "anglaises ", écrites dans le même dialecte ont pour cadre la révolte des barons en 1258-65 regroupés autour de Leicester qui était un seigneur de Montfort, descendant direct du trop fameux Simon. Leicester fut, sa vie durant, l'ennemi juré du roi Henri III. La première chanson nous a été conservée sur une bande de papier enroulée, bien d'un jongleur, et tout porte à croire que cette chanson fut colportée par des jongleurs, payés pour cela. Il s'agit en effet d'une pure œuvre de propagande où l'auteur encense le comte-mécène :

Il est apelé de Monfort :

Il est el mond et si est fort,

Si ad grant chevalerie ;

Ce voir et je m'acort,

Il eime dreit et het le tort,

Si avera la mestrie. (st. 7, v. 37 sq.) ) $^{37}$

chanson ${ }^{38}$ est, pour sa part, consacrée au regret du comte, mort à la bataille en 1265 ; il s'agit d'un planh traditionnel sur la disparition d'un grand et ses effets politiques. Plus original est le schéma de la chanson avec son refrain de six vers :

Ore est ocys

La flur de pris

Que taunt savoit de guere,

Ly quens Monfort ;

Sa dure mort

Molt en plorra la terre. (refrain p. 204)

Sa structure irrégulière, qui l'a parfois fait considérer comme un lai, son refrain et ses neuf coblas singulars de 18 vers composent un schéma formel qui est un unicum. Cette chanson politique transcende une fois encore toute classification. On notera la présence d'un texte écrit pour sa part en anglais, derrière cette même voix de jongleur : ces chansons en anglonormand représentent en effet une des dernières traces de la production française de l'île, avant l'avénement massif de la culture anglaise qui triomphe après Henri III.

La dernière chanson politique ${ }^{39}$ que j'évoquerai nous ramène en des terres connues. Elle déplore la prise de Namur en 1258 par Henri III le Blond comte de Luxembourg qui avait mis à profit une révolte de cette ville contre sa comtesse, Marie de Brienne, femme de l'empereur Baudouin de Constantinople. Cet événement eut des retombées en Champagne, terre d'où Marie était originaire, ce qui explique que les troupes venues à sa rescoussse aient compris des Champenois. Le Ménestrel de Reims accorde, lui aussi, un gros chapitre à cette même affaire ${ }^{40}$. La chanson nous est parvenue incomplète, elle fut sans doute composée par un jongleur ou un ménestrel au service d'un des seigneurs de Champagne de la troupe. Elle vitupère la lâcheté des Flamands, vaincus à Bouvine jadis et qui ne valent pas mieux «qu'un vieux peigne ». Ce texte est un nouvel exemple de male chanson.

Cahiers de recherches médiévales, 11 | 2004 
Nous entrons dans un autre monde avec le groupe bien délimité des satires écrites à Arras qui sont des chansons politiques, elles aussi, dans la mesure où les jongleurs qui les composent se font les porte-paroles des intérêts des clercs en face des bourgeois enrichis par l'usure et agrippés à leurs privilèges ${ }^{41}$. Les insultes ici se pratiquent ouvertement, nommément, en des textes fort irrévérencieux qui passent en revue tous les notables pour les étriller, en revendiquant la liberté lyrique du poète. Un brin d'irréligion pimente même le burlesque, quand on voit Dieu se laisser attirer par la renommée littéraire d'Arras, école où l'on enseigne tout ce qui est bon et utile, car Dieu espère ainsi faire passer la grosse fièvre quarte dont il souffre. Beau prétexte pour montrer l'ennui faramineux du Créateur devant les exposés scientifiques et sa délectation à écouter des chansons, mais aussi à rire à gorge déployée des clowneries de Jehan Bretel. D'autres chansons, tout aussi engagées, se déroulent à la façon du planh sur la cité d'Arras, si totalement déchue :

Arras toi qui fus jadis

La dame sans conteste

De ce pays,

Tu es confondue

Trahie, vendue

Haïe $[. . .]^{42}$

Mais la dérision et le brulesque triomphent avec le vers refrain qui clôt chaque strophe de déploration d'un Je te dirai gnif/gnaf/gnouf, etc au gré de la rime, qui sonne comme un ultime impertinence. Telle autre, dirigée sans doute contre un fait récent, glose sur l'amour des vieux ou des vieilles, sentiment par trop ridicule. Au vieux les pensées de mort, au jeune les douceurs d'amour. Le jeune a de longues années devant lui pour le repentir, les vieux sont déjà sur le chemin du cimetière, ce qui permet de dresser une longue liste (jubilatoire?) de notables arrageois partis prématurément... On le voit, la morale n'est pas des plus hautes... Tous ces textes fourmillent de données très ponctuelles qui sont un merveilleux casse-tête pour les érudits. À Arras, la satire est des plus bavardes. Les chansons comprennent une quantité insolite de couplets; cela explique que le nombre de ces chants soit finalement assez réduit, très vite leur fut préféré le dit à rimes plates qui ne connaît plus les limites imposées par la strophe, le schéma formel, le retour des rimes et la mélodie... Nous sommes sur le second versant du XIII ${ }^{e}$ siècle.

À ce stade du recensement, il ressort que la chanson politique a dû être considérée le plus souvent comme une simple outil, amusant et efficace. Il est probable que presque toutes ont péri et que seules des circonstance exceptionnelles ont sauvegardé quelques textes : la rivalité sourde de la Cour contre les grands mécènes du temps, jalousie vite étouffée par la dévotion née autour du monarque, une campagne poétique menée contre un poète notoire, une émulation arrageoise des jongleurs devant la culture trouveresque, ou encore une habitude transportée de façon très provisoire outremanche. Ces poésies empruntent à toutes les thématiques, se font satires, planh, utilisent ou boudent le refrain, empruntent un temps le dialogue de la tenson, le schéma d'un Grand Chant célèbre ou innovent en allongeant démesurément la strophe, en recourant à la facture simplifiée du chant le plus populaire avec son refrain et ses coblas singulars. En bref, il serait bien délicat de trouver des critères susceptibles de les réunir et les identifier, si ce n'est qu'elles parlent de politique et d'histoire et qu'elles le font par le chant, la strophe et la rime. Une extrême liberté semble leur avoir été concédée, sans doute assortie à leur peu de valeur intrinsèque. 

les poésies de circonstance qu'il leur est arrivé de composer. Parfois le plus convaincu de la réussite de l'œuvre était le poète lui-même : c'est le cas de Philippe de Novare qui a pris grand soin de recopier dans ses Mémoires les serventois qu'il avait composés à Chypre pour que l'oubli ne les recouvre pas. Mis à part les détails historiques toujours utiles et la personnalité de Philippe, attrayante, ces textes n'en méritaient peut-être pas tant. Ajoutons que le modèle avoué de l'auteur est le sirventes que sa grande culture lui fournit aisément.

Le terme serventois appliqué à la chanson du roi Thibaut «Deus est ensi comme li pellicanz $»^{43}$ est venu de la plume non de l'auteur, mais de ses rubricateurs. La pièce a été recopiée au même titre que toutes celles du trouvère, soigneusement conservées à son époque où sa valeur était déjà bien établie, comme elle le fut par la suite, en témoigne ce fin connaisseur qu'était Dante. La culture du roi poète est un fait bien connu et ce fils d'une Navarraise, cousin d'une princesse de Castille, ne pouvait ignorer la poésie d'oc. Mais comme tous les grands poètes, l'usage qu'il fait des œuvres contemporaines s'apparente à celui de l'intertexte. En tant que musicien, il ne s'est pas davantage astreint à reprendre les mélodies d'un autre, sauf lorsque cela l'arrangeait dans tel ou tel jeu parti. Les chansons de croisade de Thibaut sont plus consacrées à la saveur douce-amère d'un amour que l'on quitte qu'à la célébration des prouesses. Outre-mer, il cultive la nostalgie de Remenbrance si, deçà, il traite de cendreus et de morveus ceux qui hésitent à partir. De même, il use de la critique en général et en particulier: les barons ne sont pas toujours des preudommes et le siècle est plein de papelarts; l'excommunication de Fréderic II se lit à la lumière des prophéties de Merlin comme une belle sottise des clercs qui ont oublié leurs sermons pour s'en aller en guerre. Dans ses textes politiques comme dans ses satires, le poète se révèle identique à lui-même, avec sa lyrique somptueuse, débordante d'images et sa culture profane de grand seigneur à la riche bibliothèque. Le monde a bien sa place, quoique restreinte, en son art mais le monde qu'il y construit, pour notre fascination, est surtout celui de son univers intime où la maîtrise technique se laisserait presque oublier dans sa perfection.

Mais puisque déjà à cette époque, "en France, tout finissait par des chansons ", je terminerai sur une note plus divertissante avec cette chanson que Bédier appelle « un petit poème héroïco-comique, une sorte de serventois ». Ce texte fut écrite sur un mode pseudo-épique par un trouvère facétieux, afin de railler un abbé aux mœurs plutôt brutales, alors à la tête de Saint-Seine ${ }^{44}$, sur la frontière champagno-bourguignonne. Le bon abbé avait en ses terres des adversaires laïcs tout aussi remontés que lui et la petite guerre se solda par une véritable razzia qu'opérèrent le bon abbé et ses sbires sur les villages alentour à quinze lieues à la ronde. Ce fait minuscule nous est racontée avec une verve bouffonne en laquelle on avait cru reconnaître la plume du joyeux Colin Muset; en réalité, comme pour un vrai sirventes, le poète a utilisé le schéma et la musique du poème de Colin «Devers Chateauvillain». Puisque la procession du bon abbé Ponce date du milieu du règne de saint Louis, l'Anonyme et Colin, contemporains et quasi voisins, étaient sans doute des compères en l'art de railler. On notera l'aspect sautillant et guilleret que donne au texte le mètre court hexasyllabique ${ }^{45}$ :

De la procession

Au bon abbé Poinçon

Me covient a chanter :

Hons de religion

Ne fist mais tel pardon 
Par son païs aler!

Tout a fait agaster

Et tout mis a charbon.

S'il ne fust si proudom,

Il ne l'osast panser.

Il y aurait encore beaucoup à dire, assurément, pour compléter ces remarques, à la fois trop rapides et trop allusives, sur la chanson politique ou la chanson d'histoire. Mais il n'était que justice de rajouter sur la palette de nos trouvères cette couleur particulière, si souvent oubliée des critiques, que le «monde comme il va» pouvait parfois lui donner.

\section{NOTES}

1.Suzanne Thiolier-Mejean, La poétique des troubadours, trois études sur le sirventes, ( Conclusion générale », p. 395, «L'impérieuse nécessité du chant »), Centre d'enseignement et de recherche d'oc, Paris, 1994.

2.Toute cette analyse s'appuie sur le livre ci-dessus cité de S. Thiolier, ch. II, « un devenir incertain : le vers, le sirventès et la poésie d'oïl », p. 285 sq. et sur Martin Aurell, La vielle et l'épée, Aubier, 1989.

3.A. Jeanroy, La poésie lyrique des troubadours, Slatkine Reprints, Genève, 1973, volume 2, p. 183, (définition du sirventes « personnel »).

4.Les leys d'Amors, édition de Gatien-Arnoult, Toulouse, 1841-43; la citation provient de l'article « sirventes » du Dictionnaire des Lettres Françaises (Le Moyen Âge), nouvelle édition sous la direction de M. Zink et G. Hasenohr, Paris, La Pochothèque, 1992. 5.Édité par P. Meyer, «traités catalans de grammaire et de poétique ", Romania, VI, 1877, pp. 341-58.

6.Ou devrait passer, ce qui n'est pas, certes, le cas au Nord...

7.Principalement D. Rieger, Gattungen und Gattungsbezeichnungen der Trobadorlyrik, Tübingen, 1976, que cite abondamment l'ouvrage ci-dessus évoqué de S. Thiolier. Cf. aussi son article sur le sirventes dans le GRLMA, II, 1 fascicule 4, pp. 9-61.

8.P. Bec, La lyrique française au Moyen Âge, contribution à une typologie des genres poétiques médiévaux, deux volumes (études et textes), Paris, Picard, 1977.

9.Wace, Le roman de Rou, édité par J. Holden, Paris, SATF, 1970-1973 (v. 53).

10.Le roman de Thèbes, édité par Raynaud-Delage, Paris, Champion, CFMA, 1966.

11.Cité dans l'édition de J. Barrois, Paris, 1842 (Roman des douze pairs de France).

12.Moniot d'Arras et Moniot de Paris, trouvères du XIII siècle, édition des chansons et étude historique de H. Petersen Dyggve, Helsinki, 1938 (RS 1835, p. 141, nXXVII).

13.Jacques Ribard, Un ménestrel du XIVe siècle, Jean de Condé, Genève, Droz 1969.

14.Raynaud-Montaiglon, Les deux bourdeor ribauds, Recueil général des fabliaux, Genève Reprints, 1973.

15.S. Thiolier, op. cit., p. 313. 
16.Cf. M.-G. Grossel, « Entre rire et chastoiement, les chansons satiriques et bachiques (remarques sur un genre à (re)définir) », «Pur remenbrance », Mélanges en souvenir de Wolfgang Spiewok, Reineke Verlag, 2001, p. 171-196.

La différence établie ici entre «satiriques » et "politiques » est donc très pragmatique : d'une part, je garde «satiriques » pour les textes édités par Jeanroy-Långfors sous cette épithète dans leur célèbre Chansons satiriques et bachiques du XIII ${ }^{e}$ siècle, Paris, Champion, 1974.

D'autre part, je préfère ranger sous le label «satiriques" celle qui traitent davantage de questions morales (décadence $\mathrm{du}$ siècle, dévalorisation d'amour voire perversion des « ordres »...) et sous le label " politiques » celles qui traitent d'événements « historiques », même s'il ne s'agit que du décès d'un riche arrageois... Le choix, fatalement contestable, reste résolument thématique. Ainsi je n'étudierai pas ici la chanson de Jacques de Cysoing, RS 1205, $\mathrm{n}^{\circ} 1$ de l'édition Jeanroy-Långfors (mot serventois dans l'envoi au comte de Flandre) que je laisse, après les deux grands savants, sous sa rubrique "satirique contre le siècle "; rappelons en passant que Jacques de Cysoing, documenté deuxième moitié du XIII ${ }^{\mathrm{e}}$ siècle, fait partie des tout derniers trouvères.

17.P. Bec, op. cit., volume I, p. 150 sq.

18.J. Bédier et P. Aubry, Chansons de croisade, Genève, Slatkine Reprints, 1974.

19.P. Bec, op. cit., volume I, p. 152.

20.Mise à part la première, mais pas dans le domaine lyrique comme je l'ai remarqué plus haut.

21.On peut difficilement dire que le Châtelain de Couci est un poète qui célèbre la quatrième croisade, puisqu'il se contenta de partir (et mourir), non vraiment de participer à ce détournement notoire. Quant à sa participation à la troisième croisade (1190), choix retenu par Bédier-Aubry, elle est devenue peu vraisemblable depuis que les travaux de D. Barthélémy, Les Deux âges de la seigneurie banale de Coucy, Paris, Publications de la Sorbonne, 1984, ont considérablement affiné (et rajeuni) les dates de la vie du trouvère.

22.Chanson 1, Bédier-Aubry, op. cit., p. 8 sq.

23. Chanson 2, p. 20 sq.

24.Toutes les chroniques qui l'évoquent s'accordent à le décrire comme un homme railleur et irascible, autant que rempli de bravoure.

25. Chanson 4 , p. 45 sq.

26. Chanson 7 , p. 75 sq.

27. Chanson 9, p. 97 sq.

28. On trouve de nettes réminiscences de ces vers (presque des citations) aussi bien dans Fouques de Candie que dans la version continentale I (d'origine probablement champenoise) de Beuve de Hanstone : cf. M.-G. Grossel, « Onques tant ne fit por Foucon Anfelixe Com ie por vos... Composition et romanesque dans Fouques de Candie d'Herbert le Duc », Plaist vos oïr bone cançon vallant? (Mélanges offerts à François Suard). Coll. UL 3, Lille 1999, p. 351-361 et le colloque d'Amiens Héros épiques, héros romanesques de mars 2004 : « De la geste à l'aventure : le héros dans la chanson de Beuve de Hanstone » (version continentale 1 « champenoise »).

29.On rappellera l'exemple si connu de Renart de Dampierre qui mit trente ans à revenir de la quatrième croisade.

30.Chanson 21, p. 231 sq.

31. Chanson 20, p. 221 sq.

32. Chanson 23, p. 251 sq.

33.Gaston Paris, « La chanson composée à Acre », Romania 22, 1897, p. 541-42. 
34.Sur tous ces points, cf. Marie-Geneviève Grossel, Le milieu littéraire en Champagne sous les Thibaudiens, Paradigme, 1994.

35.Les chansons politiques sont citées d'après l'édition Leroux de Lincy, Recueils de chants historiques français depuis le XII jusqu'au XVI ${ }^{e}$ s., Paris, 1841. Ici p. 165 sq.

36.Édition Leroux de Lincy citée, p. 176 sq.

37.Éd. cit. p. 200 sq.

38.P. 204 sq.

39.Éd. cit. p. 211 sq.

40.Cf. M.-G. Grossel, Les récits d'un Ménestrel de Reims, traduction et notes, Presses Universitaires de Valenciennes, 2002.

41.Bien qu'il participe des mêmes cercles régionaux, Alard de Cans, dont on cite souvent, dans les comparaisons oc/oïl la chanson « E serventois, arrière t'en revas », est vraiment un cas à part ; cet artésien de haut rang (il sortait de la famille des Croisilles) documenté vers 1235, a écrit sa chanson RS 381 (qui mériterait d'ailleurs plus justement le titre de Congé) « sur » le planh de Gaucelm Faidit déplorant la mort du roi Richard ; il lui emprunte le schéma formel et la mélodie comme l'a établi depuis longtemps H. Spanke (G. Raynauds Bibliographie der altfranzösischen Lieder, Leiden, Brill, 1955, p. 82). Il semble évident que le terme « serventois » a été lui aussi choisi dans ce cas très particulier pour souligner le choix opéré par le poète. Quant à se demander si le rapport entre le planh initial et l'adieu aux « bonnes gens d'Arras » - et tout particulièrement au compère Copin Doucet élu messager près de la dame - n'est pas un brin ironique, je laisse à d'autres le soin d'en juger ! On lira RS 381 dans l'édition de H. Spanke, Eine altfranzösische Liedersammlung, Halle, $1925\left(=\mathrm{n}^{\circ} 40\right)$.

42.La chanson est citée d'après l'édition des Chansons de trouvères, Chanter m'estuet, édition des textes et mélodies de S. Rosenberg et H. Tischler, Lettres Gothiques, Paris, 1995, p. 267.

43. Les chansons de Thibaut de Champagne, roi de Navarre, édition critique d'A. Wallensköld, Paris, SATF, 1925 (il s'agit de RS 273, n LVI de l'édition citée).

44.Aujourd'hui Saint-Seine-l'Abbaye.

45.Les chansons de Colin Muset, éditées par J. Bédier, Paris, Champion, 1938, RS 1881, chanson, XXI, p. 37 et A. Longnon, « De la procession », Romania, 30, 1901, p. 198-212.

\section{AUTEUR}

\section{MARIE-GENEVIĖVE GROSSEL}

Université de Valenciennes et du Hainaut-Cambrésis 